\title{
A stern view of gastrulation
}

Gastrulation: From Cells to
Embryo
Edited by Claudio Stern

Gastrulation is a process common to nearly all metazoan organisms during which an embryo with distinct tissue layers develops out of a seemingly unstructured assembly of cells referred to as 'blastula'. This is achieved by a highly coordinated series of cell movements involving cell-cell interactions and changes in cell fate, variations of which can also be found to occur during later stages of embryogenesis in many organisms. The study of gastrulation, therefore, not only attracts researchers interested in gastrulation per se but it has also become the subject of choice for a steadily increasing number of researchers who wish to study basic molecular mechanisms and developmental principles in a relatively simple developmental context.

In the first half of the last century, classical studies in different model systems yielded important findings about the cellular rearrangements and cell movements underlying gastrulation. By contrast, investigation of the molecular control of gastrulation is still a relatively young field of research where many of the underlying principles are still being intensely debated. Considering the somewhat preliminary stage of molecular research in this area, one might ask if it is a little early to devote a textbook to this topic. Nonetheless, a new book called Gastrulation: From Cells to Embryo, and edited by Claudio Stern, provides a much needed integrative view of this complex field.

This book deals with the apparent complexity of gastrulation by structuring its content into five distinct parts: Embryology, Cellular Events, Molecular Biology, Evolution and Open Questions. This arrangement allows the reader to appreciate gastrulation from different perspectives and to gain a general understanding of the common molecular and cellular principles underlying gastrulation.

The coverage of key topics within each chapter is comprehensive and well illustrated with numerous figures and the effort made to keep the styles of writing and illustrations consistent between chapters is helpful. The different chapters, each one written by experts in the field, not only give us the facts, but also clearly communicate the enthusiasm that the authors have for their topics. Although the content is sometimes very detailed, the book is quite didactic and even the beginner will be

Irinka Castanon-Ortega and Carl-Philipp Heisenberg

Are at the Max-Planck-Institute of Molecular Cell Biology and Genetics.

Pfotenhauerstrasse 108, 01307 Dresden, Germany.

e-mail: castanon@mpi-cbg.de and heisenberg@mpi-cbg.de easily drawn into this subject. In the first part of the book, gastrulation movements in a wide-range of metazoan organisms are explained, and it describes not only the diversity of cell movements employed by different organisms but it also points out the major cellular rearrangements and morphogenetic movements that are conserved among them. The second part deals with gastrulation at the cellular level and discusses different modes of mesoderm internalization and migration, including how the mesoderm is induced and how embryonic asymmetry and patterning are established. In the third and most novel part of the book, recent advances in the molecular biology of gastrulation are discussed that shed light on the mechanisms by which different signalling pathways, such as Nodal, Wnt, FGF, Notch and BMP, as well as transcription factors and extracellular components control patterning and morphogenesis during gastrulation. The fourth part considers gastrulation from an evolutionary perspective by comparing gastrulation between fossil and more recent embryos, whereas the fifth and last part of the book, written by Lewis Wolpert, provides a much-needed overview of what is known about gastrulation, and the challenges that lie ahead.

The strength of this book, which is a description of gastrulation in many organisms and from a wide variety of perspectives, could also be viewed as its main weakness. Compared with other more established fields in biology, the basic principles and mechanisms of gastrulation are still controversial and need detailed illustration. This overwhelming amount of information might be difficult for the non-expert wishing to gain a general overview on gastrulation without the risk of drowning in details. This difficulty can be seen in the chapter on embryology, where model systems such as crustaceans, molluscs or rabbit do not yet provide sufficient information to substantially advance our general understanding of gastrulation, despite their long-term prospects of success. The incorporation of this information does not add important new concepts to that derived from more extensively studied model organisms, such as frogs, zebrafish and flies, and consequently does not contribute to the 'big picture'.

This book - as it is the case with most textbooks — cannot be considered as the ultimate source of recent work. But it nicely summarizes current research in the field and it will be an invaluable resource for laboratories interested in gastrulation. This first textbook solely dedicated to gastrulation might perhaps be a little premature with respect to the current knowledge on molecular processes in gastrulation, but it is certainly a useful resource for advanced undergraduates, graduate students and other new entrants into this field.

New in hardback

Live Cell Imaging: A laboratory manual

Edited by Robert D. Goldman and David L. Spector

Cold Spring Harbour Laboratory Press, 2005. \$250/£170 